\title{
Detecting quantitative trait loci affecting beef tenderness on bovine chromosome 7 near calpastatin and lysyl oxidase
}

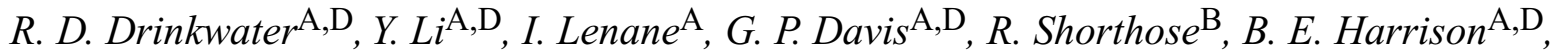

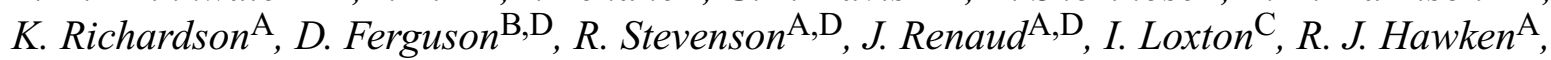 \\ M. B. Thomas A , S. Newman ${ }^{\mathrm{A}, \mathrm{D}}$, D. J. S. Hetzel ${ }^{\mathrm{A}, \mathrm{D}}$ and W. Barendse $\mathrm{A}, \mathrm{E}$ \\ ${ }^{\mathrm{A}}$ CSIRO Livestock Industries, Level 5 Queensland Bioscience Precinct, \\ 306 Carmody Road, St Lucia, Qld 4067, Australia. \\ ${ }^{B}$ Food Science Australia, Cannon Hill, Qld 4170, Australia. \\ ${ }^{\mathrm{C}}$ Queensland Department of Primary Industries and Fisheries, Rockhampton, Qld 4701, Australia. \\ ${ }^{\mathrm{D}} \mathrm{CRC}$ for the Cattle Industry (Beef Quality), Armidale, NSW 2350, Australia. \\ ${ }^{\mathrm{E} C o r r e s p o n d i n g}$ author. Email: bill.barendse@csiro.au
}

\begin{abstract}
From a study of 3 large half-sib families of cattle, we describe linkage between DNA polymorphisms on bovine chromosome 7 and meat tenderness. Quantitative trait loci (QTL) for Longissimus lumborum peak force (LLPF) and Semitendonosis adhesion (STADH) were located to this map of DNA markers, which includes the calpastatin (CAST) and lysyl oxidase (LOX) genes. The LLPF QTL has a maximum lodscore of 4.9 and allele substitution of approximately 0.80 of a phenotypic standard deviation, and the peak is located over the CAST gene. The STADH QTL has a maximum lodscore of 3.5 and an allele substitution of approximately 0.37 of a phenotypic standard deviation, and the peak is located over the LOX gene. This suggests 2 separate likelihood peaks on the chromosome. Further analyses of meat tenderness measures in the Longissimus lumborum, LLPF and LL compression (LLC), in which outlier individuals or kill groups are removed, demonstrate large shifts in the location of LLPF QTL, as well as confirming that there are indeed 2 QTL on bovine chromosome 7. We found that both QTL are reflected in both LLPF and LLC measurements, suggesting that both these components of tenderness, myofibrillar and connective tissue, are detected by both measurements in this muscle.
\end{abstract}

\section{Introduction}

The process of finding quantitative trait loci (QTL) for key livestock traits has matured such that results are now being confirmed and diagnostic DNA markers for traits are starting to be offered (e.g. Barendse 1997, 2001; Page et al. 2002). However, this maturity belies the difficulty of locating and then identifying the QTL, particularly when there are likely to be several QTL on a chromosome affecting the trait, and when the confirmation in independent studies, of the existence of the QTL, is lacking. In this study we report a detailed analysis of QTL for meat tenderness that illustrates some of the difficulties that can arise in trying to narrow down the location of a QTL in the shifting sands of genetic recombination.

The expression of beef tenderness is a function of many factors (reviews by Ferguson et al. 2001; Oddy et al. 2001) that include the genotype and production history of the animal, post-slaughter management of the carcass and the cooking conditions of the meat, which could increase the difficulty of locating the QTL accurately. Intrinsic attributes within muscle that have a considerable influence on meat tenderness are the rate and extent of post-mortem tenderisation, which is governed by the interaction between the proteolytic calpains and their inhibitor calpastatin, the degree of collagen crosslinking in connective tissue, some of which is related to age of the animal, and the sarcomere length, which can be affected by processing of the animal, such as occurs with cold shortening. The establishment of crosslinks within collagen is initiated by the enzyme lysyl oxidase (Kuypers and Kurth 1995). Both of the enzymatic attributes can be quantified directly via biochemical assays or indirectly via the changes in the textural properties of the meat. Thus, tenderness can be measured in different ways, which could give different locations for QTL, and which could affect the location of QTL if there were several different QTL affecting meat tenderness on the same chromosome.

Initial studies (Chung et al. 1999; Lonergan et al. 1995; Palmer et al. 1997) of DNA variation at the calpastatin gene reported no association to meat tenderness, although it was well documented that differences in calpastatin activity at the same levels of calpain activity are related to meat tenderness (e.g. Koohmaraie 1996; Whipple et al. 1990). Here we report data locating QTL to bovine chromosome (Bta) 7 in the vicinity of the calpastatin (CAST) and lysyl oxidase (LOX) genes studied in 3 large half-sib pedigrees with cross-bred 
Charolais $\times$ Brahman sires. This breed cross was chosen because of the expected differences in meat tenderness between Charolais and Brahman. The data show the presence of 2 QTL for meat tenderness on the same chromosome. However, depending upon the analysis that is performed, the QTL are shifted towards the centromere of the chromosome, and we explore some of the limitations and ramifications of this for locating QTL.

\section{Materials and methods}

Cattle samples

For linkage mapping of QTL, the CBX samples described by Davis et al. (1998) were used. In short, there are 3 sire families of Charolais $\times$ Brahman bulls with a total of 599 offspring, 2 of the sires share a dam, and the dams are of the Belmont Red breed (AXBX Afrikander Hereford-Shorthorn Brahman Hereford-Shorthorn), all bred and raised at the Belmont Research station and then measured for at least 90 phenotypic traits. Not all animals have joint phenotypes and genotypes for all traits and all loci.

Blood was collected into EDTA tubes (Becton Dickinson, North Ryde, NSW, Australia) and processed to DNA using standard methods (e.g. Barendse et al. 1993, 2004; Maniatis et al. 1982). DNA was diluted to about $50 \mathrm{ng} / \mu \mathrm{L}$ for the working stock and plated into a 96 well format.

\section{Meat tenderness measurements}

The cattle were transported to the Central Queensland abattoir near the Belmont Research station. The cattle were slaughtered in groups of 30-40 between 1992 and 1994. On the day after slaughter, a sample of the Longissimus lumborum (LL) and the Semitendonosis (ST) was removed and frozen at $-20^{\circ} \mathrm{C}$. The frozen samples were transported to the Food Science Australia Cannon Hill Laboratory for meat tenderness evaluation. The samples were thawed at $5^{\circ} \mathrm{C}$ for $48 \mathrm{~h}$. The samples were trimmed of fat and epimysial tissue and reduced to a size of $250 \mathrm{~g}$ for cooking. The samples were then cooked for $1 \mathrm{~h}$ in an $80^{\circ} \mathrm{C}$ water bath. After cooking the samples were cut and prepared and the tenderness measurements of LL peak force (PF) and compression were obtained, and $\mathrm{ST}$ adhesion $(\mathrm{ADH})$ and compression were made according to the procedures described by Bouton et al. (1971) and Bouton and Harris (1972). A peak force measurement represents more the myofibrillar component of toughness (and therefore reflects changes owing to postmortem proteolysis), while the adhesion and compression measurements represent more the connective tissue toughness (and, therefore, reflects collagen cross-linking) (Kuypers and Kurth 1995). The tests were performed by an Instron Universal Testing machine.

\section{DNA polymorphisms}

Eight DNA microsatellites and 2 single strand conformational polymorphisms were used for the linkage map of bovine chromosome 7. The gene order on the map, BM7160-RM012-RM006LOX-CSSM029-BM1853-RASA-ILSTS006-CAST-INRA053, was taken from a combination of the published reference maps (Barendse et al. 1997; Kappes et al. 1997). The primer sequences of these DNA markers are presented in Table 1. Microsatellite genotypes were separated on an ABI 373A semi-automated DNA sequencer and the genotypes curated and scored using the Genescan and Genotyper software following the manufacturer's instructions (Applied Biosystems Inc., Foster City, CA) between 1994 and 1999. In addition to these microsatellites, a single nucleotide polymorphism for the calpastatin and for the lysyl oxidase gene was identified, the former in the $3^{\prime}$ untranslated region and the latter in the fifth intron. These were genotyped by single strand conformational analysis using polyacrylamide gel electrophoresis and autoradiography using published methods (Barendse et al. 1993; Orita et al. 1989).

Table 1. Primers and probes for DNA microsatellites and single nucleotide polymorphisms

\begin{tabular}{|c|c|c|}
\hline Locus & DNA sequence & Reference \\
\hline \multicolumn{3}{|c|}{ SNP MGB assays } \\
\hline CAST3U2 & TGTTCTGATTTCTCATGACCCCT & Barendse (2001) \\
\hline CAST3D2 & TTATGACTGACTTATAAACAACAACCCA & \\
\hline CASTSNP1-1 & 6FAM-TCCTCTTGGACTTGTGGAC & \\
\hline CASTSNP1-2 & VIC-TCCTCTTAGACTTGTGGAC & \\
\hline LOXESNPU3 & GGTCCTGCAAACAATTATACATCTTC & Barendse (2001) \\
\hline LOXESNPD3 & GAAGGAGGCTGGATCTTTTTCC & \\
\hline LOXESNP2-1 & 6FAM-CTAGATATATTTAAAAAATTCT & \\
\hline LOXESNP2-2 & VIC-CTAGATATATTAAAAAAATTCT & \\
\hline \multicolumn{3}{|c|}{ Microsatellite assays } \\
\hline BM1853F & AGCCTTTTGTAGGTGTTCATTG & Bishop et al. (1994) \\
\hline BM1853D & ATGGGGTTGCAAAGAGTCAG & \\
\hline BM7160F & TGGATTTTTAAACACAGAATGTGG & Stone et al. (1995) \\
\hline BM7160D & TCAGCTTCTCTTTAAATTTCTCTGG & \\
\hline CSSM029F & GCTCCATTATGCACATGCCATGCT & Moore et al. (1994) \\
\hline CSSM029D & CGTGAGAACCGAAAGCACACATTC & \\
\hline ILSTS006F & TGTCTGTATTTCTGCTGTGG & Brezinsky et al. (1993) \\
\hline ILSTS006D & ACACGGAAGCGATCTAAACG & \\
\hline INRA053F & AAAGTCAGATACAACTGAGTGAC & Vaiman et al. (1994) \\
\hline INRA053D & AATCACCAGAAATTCACTTCACC & \\
\hline RASAF & TTCCGCTTTAGTGCAGCCAGGAAGT & Moore et al. (1992) \\
\hline RASAD & TCCCCGGGTTACGGGAAGAGAAGTG & \\
\hline RM012F & CTGAGCTCAGGGGTTTTTGCT & Kossarek et al. (1994) \\
\hline RM012D & ACTGGGAACCAAGGACTGTCA & \\
\hline RM006F & CTACAATATCTGGTCACTGGA & Barendse et al. (1994) \\
\hline RM006D & GATCACCATATTTATGAGATGG & \\
\hline
\end{tabular}


Specifically, CAST was analysed on a $6 \%$ non-denaturing polyacrylamide gel (59:1 acrylamide: bis-acrylamide containing 5\% glycerol in $0.5 \times$ TBE (Tris-Borate EDTA, $\mathrm{pH} 8.3$ ) at $4 \mathrm{~W}$ for $16 \mathrm{~h}$ before the gels were dried and $\mathrm{x}$-ray film exposed. The fragment containing the LOX polymorphism was first digested with HinF1 before the fragments were separated on the same kind of SSCP gel as above. Both of these SSCP (CAST3-A84G and LOXE-A183T) have been sequenced and have been replaced with high throughput MGB Taqman assays (Table 1; Barendse 2001).

Recombinants in the linkage map were visualised using crimap and close double recombinants were removed as erroneous. Distances were then calculated for this map and then used as input for the Animap program (Georges et al. 1995).

Analysis between DNA polymorphisms and meat tenderness

For the linkage analysis, the phenotypes were pre-analysed so that a residual meat tenderness value could be analysed using a maximum likelihood approach. The model was:

Trait $=$ mean + contemporary group + sire + dam's selection line + dentition + age within contemporary group + sequence within contemporary group.

The contemporary group is a combination of cohort (year of birth), sex (steer or heifer), and slaughter group (6 for 1992, 9 for 1993, and 6 for 1994). Age was fitted within contemporary group since there was a range of about 40 days of age within each contemporary group. Initial analyses showed that the sequence of slaughter within a contemporary group was an important source of variation. After fitting the variables, those that were non-significant were removed from the model and residuals obtained for all individuals with records. They were then matched up with the genotype data for the QTL analyses. The analysis of the half-sib data was performed using the Animap program, which gives a maximum likelihood estimate of the linkage and is expressed as a lodscore.

As we found a QTL in more than 1 place on the chromosome, this offered us the opportunity to examine the linkage analysis from several different aspects, including alternative methods of analyses. We tried an alternative scheme for generating residuals as well as an alternative software for calculating maximum likelihood estimates of linkage. The alternative model was:

Trait $=$ mean + contemporary group + sire + dam's selection line + dentition + age within contemporary group + sequence $x$ contemporary group.

The major difference is that age and sequence were treated as covariates and not nested as in the first model. The residuals were analysed using a version of the linkage analysis software by Kerr et al. (2005). We restricted the re-analysis to the Longissimus lumborum data since we wished to view the impact within a specific tissue and so avoid the issues of precision of localisation in different tissues and different methods of measuring tenderness. We focused on Longissimus lumborum since it is much more valuable as a cut than the Semitendonosis, noting that the LL compression is not as good an estimator of connective tissue toughness as either ST compression or ST adhesion. The software of Kerr and Henshall provides output as a likelihood ratio not a lodscore and the threshold for significance was determined by simulation of 1000 replicates. In addition to using a different model for calculating residuals and for estimating maximum likelihood, the following alternative datasets were used. These are called V1, V2 and V3. V1 is where animals with LLPF greater than 2 standard deviations from the mean were removed, that is, those with LLPF $>7.5 \mathrm{~kg}$ peak force, leaving a sample of 512 . V2 is where 3 kill groups with anomalously high mean LLPF values were removed, leaving a sample of 486 . V3 is a combination of V1 and V2, leaving a sample of 451 animals with joint phenotypic and genotypic data.

\section{Results}

The genetic linkage map was pter-BM7160-13-RM012-10RM006-10-LOX-15-CSSM029-16-BM1853-6-RASA-9ILSTS006-6-CAST-4-INRA053-qter with distances in Haldane centiMorgans (cM) for these genotypes.

Using the first model and the animap software, a QTL for LLPF was mapped to chromosome 7 with a maximum lodscore of 4.9 with a peak at the CAST3-A84G polymorphism (Fig. 1). Only 1 of the pedigrees, family 3 , shows segregation for the QTL. The size of effect calculated using this model indicates an allele substitution effect of 0.8 of a phenotypic standard deviation.

A QTL for STADH was also mapped to chromosome 7 with a maximum lodscore of 3.5 with a peak at the LOXE-A183T polymorphism (Fig. 1), but this QTL does not overlap with the QTL for LLPF. Two of the 3 pedigrees appear to be segregating the QTL. The size of effect calculated using this model indicates an allele substitution effect of 0.36 of a phenotypic standard deviation. The ST compression data do not show a statistically significant association, but there is some evidence (data not shown) for segregation in family 1 only, not enough to be statistically significant.

The effects of removing some of the data are shown in Table 2. Removal or control of the outliers within the LLPF dataset as defined above resulted in an improvement in the normality of the data but they were still significantly skewed. Removal of outliers had a slight effect on the mean LLPF for the sample, reduced the standard deviation, but had little effect on the distribution of LLC (Table 2), which shows minimal departure from normality.

The alternative analysis shifts the location of the LLPF peak along the chromosome by about $40 \mathrm{cM}$, placing the peak between the LOX and CAST genes (Fig. 2). However, removing the outliers, dataset $\mathrm{V} 1$, shifts the peak an

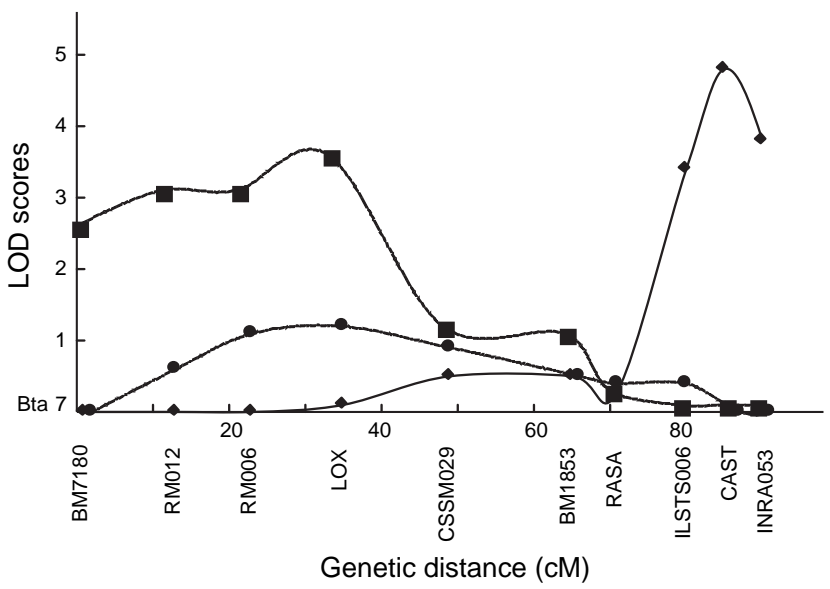

Fig. 1. Lodscore plot of the LLPF $(\bullet$; lod $=4.93)$, STADH $(\mathbf{\square}$; lod $=$ 3.54) and STC $(\boldsymbol{O}$; lod $=1.29)$ measures of meat tenderness on bovine chromosome 7 . 
Table 2. Summary statistics for meat quality traits

Full, full dataset; V1, removal of samples with LLPF greater than 7.5; V2, removal of 3 kill groups with highest average LLPF; V3, V1 combined with V2

\begin{tabular}{|c|c|c|c|c|c|c|c|}
\hline Trait & $n$ & $x$ & s.d. & Minimum & Maximum & Kurtosis & Skewness \\
\hline \multicolumn{8}{|c|}{$L L P F$} \\
\hline Full & 564 & 5.63 & 1.47 & 3.28 & 14.53 & 5.87 & 1.77 \\
\hline V1 & 512 & 5.30 & 0.97 & 3.28 & 7.47 & -0.67 & 0.30 \\
\hline V2 & 486 & 5.47 & 1.31 & 3.28 & 14.36 & 4.93 & 1.51 \\
\hline V3 & 451 & 5.23 & 0.96 & 3.28 & 7.45 & -0.58 & 0.36 \\
\hline \multicolumn{8}{|c|}{$L L C$} \\
\hline Full & 564 & 2.16 & 0.24 & 1.53 & 2.88 & -0.29 & 0.16 \\
\hline V1 & 512 & 2.16 & 0.24 & 1.53 & 2.88 & -0.32 & 0.18 \\
\hline V2 & 486 & 2.15 & 0.24 & 1.53 & 2.88 & -0.19 & 0.21 \\
\hline V3 & 451 & 2.14 & 0.25 & 1.53 & 2.88 & -0.20 & 0.25 \\
\hline
\end{tabular}

additional $30 \mathrm{cM}$ towards the centromere. This latter shift is also a shift from segregation found in family 3 to that found in family 1, i.e. not a QTL location shift but a QTL identity shift. Removal of some kill groups, dataset V2, shows the presence of 2 peaks in the likelihood ratio plot for LLPF (Fig. 2), owing to information for both families 1 and 3 having an impact on the overall plot. The dataset V3, the combination of both forms of removal of outliers, is similar to that of V1, but with lower likelihood ratios.

However, the alternative analysis for LLC shows an obvious difference to that of LLPF because there are always 2 peaks in the likelihood ratio plot for LLC along the chromosome, and interestingly, 1 of the peaks is re-centred over the CAST gene (Fig. 3). With minor differences in peak height, the LRT plots for all of the linkage analyses shows 2 peaks, 1 centromeric to LOX and 1 over CAST, the 1 centromeric to LOX is for family 1 and the 1 near CAST is for family 3 . The peak heights for LLC are lower than for

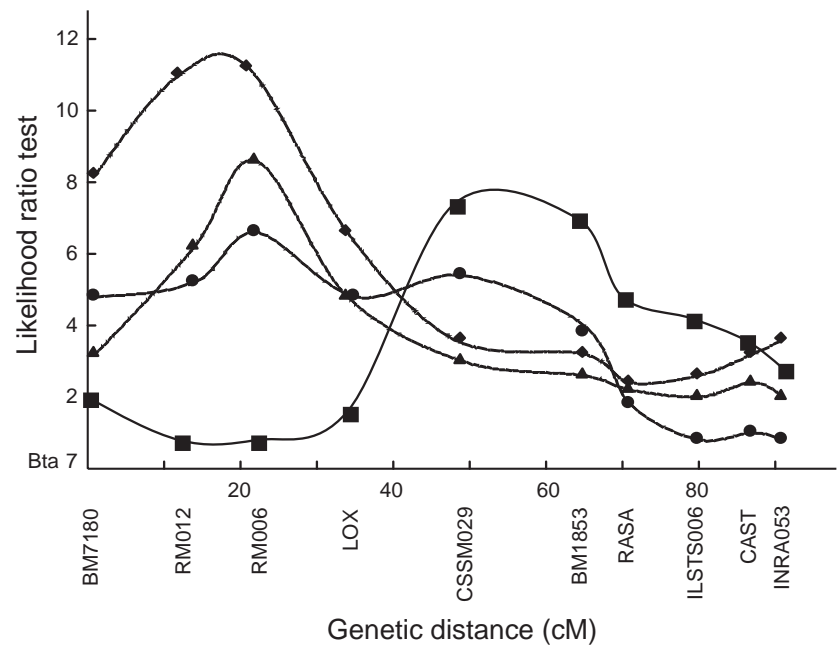

Fig. 2. Likelihood ratio plot of the LLPF measures of meat tenderness on bovine chromosome 7 with different data subsets [Full $(\mathbf{\square}), \mathrm{V} 1(\diamond)$ V2 $(\bullet)$, V3 $(\boldsymbol{\Delta})]$ to control outlier observations.
LLPF, consistent with the lower values found for ST compression (see above).

\section{Discussion}

We found statistically significant evidence for 2 QTL affecting tenderness on bovine chromosome 7 at a threshold for whole genome studies (Lander and Kruglyak 1995), with peaks near the LOX (STADH) and the CAST (LLPF) gene. Differences in protein activity for these genes and, more recently, DNA variation at 1 of these genes, has been reported to be associated with meat tenderness (e.g. Barendse 2001). The peak suggesting QTL near LOX is new, and the peak suggesting QTL near CAST contradicts some previous reports of a lack of association between CAST DNA polymorphisms and meat tenderness (Chung et al. 1999; Lonergan et al. 1995; Palmer et al. 1997). Two related conference posters (Green et al. 1996a, 1996b) at the same conference are the only other positive evidence of

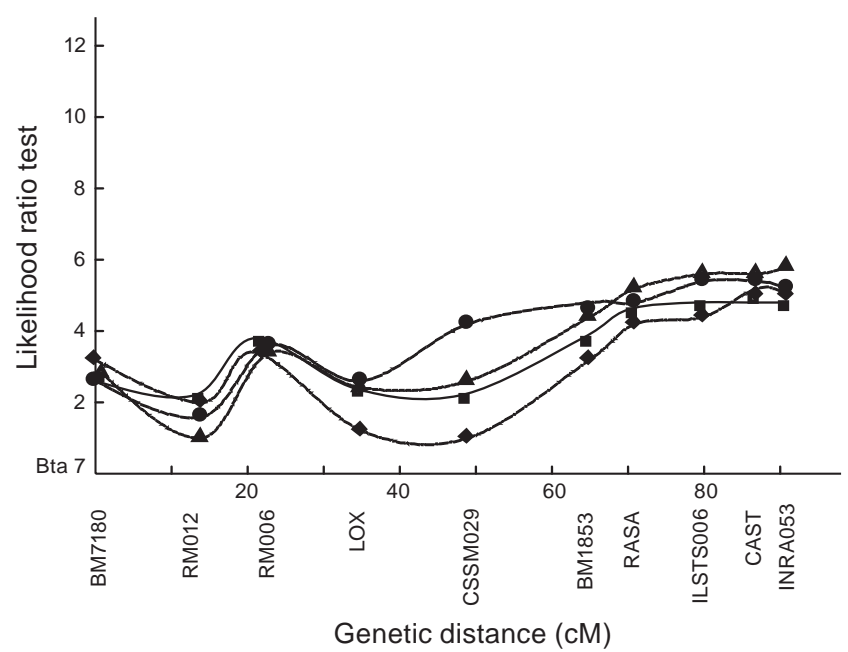

Fig. 3. Likelihood ratio plot of the LLC measures of meat tenderness on bovine chromosome 7 with different data subsets [Full $(\mathbf{\square}), \mathrm{V} 1(\diamond)$, V2 $(\bullet)$, V3 (A)] to control outlier observations. 
reported associations between CAST and meat tenderness, although these were only at $\alpha=0.05$ and so cannot be classed as evidence at the genome-level for a new linkage.

The effects of data analysis, software and method of measuring tenderness on the location and number of QTL were profound. The peak of the likely location of the QTL for LLPF shifted by $40 \mathrm{cM}$ along the chromosome merely by making a minor change to the method of calculating the residuals and by changing the exact method of calculating the maximum likelihood. This is cause for concern to all those who wish to use linkage mapping to locate QTL. These results show that performing a single kind of analysis may not be sufficient to get an accurate location. Not only should different methods and software be used, but different measurements of the trait should also be used to home in on the location of the QTL. A shift of $40 \mathrm{cM}$ represents on average about $40 \mathrm{Mbp}$ in mammals, which would contain literally hundreds of possible genes, many of which could then be candidates to contain causative mutations. Moreover, when methods disagree to this extent, choosing the better model of the 2 cannot be determined by argument but will require additional collection of data (see Barendse 2005).

The number of QTL for LLPF also changed with different analyses, but these differences served to confirm that there were in fact more than 1 QTL on the chromosome. This can be most clearly seen when the evidence for the LLPF QTL shifted from 1 family to another, which coincided with a shift of the location of the QTL along the chromosome, and in the evidence for the LLC QTL, which showed 2 peaks irrespective of the analytical method.

These analyses show that extreme phenotypic values for 1 measure can obscure the impact of 1 of the 2 networks on meat tenderness. Compression tests are usually considered to measure the degree of meat toughness contributed by connective tissue, which is partly related to the degree of cross-linking in collagen. Peak force measurements are usually considered to measure the degree of meat toughness contributed by myofibrillar differences, usually ascribed to interaction between the overall extent of post-mortem myofibrillar shortening and proteolytic tenderisation. In this analysis, the LLC linkage analysis shows both families having an impact on meat tenderness, with the genetic basis located to different parts of the chromosome. The LLC LRT plots are similar to the LLPF LRT plots when the outlier slaughter groups are excluded, which may indicate that there is cross-talk in the measurement. The LLPF analyses were affected by the inclusion of the extreme values compared to the LLC analyses, since the LLPF analyses show large changes in the LRT plots depending on what dataset is used.

In this study, the families are large enough that each could identify the segregation of the QTL but studies with much smaller numbers of offspring per family would not be able to detect segregation of the QTL by themselves. In those studies, one would need to group like families together and assume heterogeneity between groups of families. But in this flawed approach how would one then discriminate between a real heterogeneity and an arbitrary choice of families? It would be an arbitrary choice that then allows the spurious finding of a QTL. Nevertheless, the overall LRT plot would suggest that the most likely location of the QTL had shifted significantly along the chromosome. By analysing 2 completely different measures of meat tenderness, different methods of analysis, and using large pedigrees, we have been able to simplify what would otherwise have been a complicated story of QTL locations.

\section{Acknowledgments}

We thank Mike Goddard for statistical advice, and Belinda Norris and James Kijas for comments which improved the manuscript. We thank the Cooperative Research Centre for the Cattle Industry (Beef Quality) for financial support. These data were unpublished background Intellectual Property for the CAST3 SNP, which has been licenced for commercial testing as the GeneSTAR tenderness test.

\section{References}

Barendse W (1997) Assessing lipid metabolism. Patent WO9923248 Patent US6383751 (Patent Application PCT/AU98/00882). Available at http://ep.espacenet.com/ (verified 3 January 2006)

Barendse W (2001) DNA markers for meat tenderness. Patent WO02064820 (Patent Application PCT/AU02/00122). Available at http://ep.espacenet.com/ (verified 3 January 2006)

Barendse W (2005) The transition from quantitative trait loci to diagnostic test in cattle and other livestock. Australian Journal of Experimental Agriculture 45, 831-836. doi:10.1071/EA05067

Barendse W, Armitage SM, Ryan AM, Moore SS, Clayton D, Georges M, Womack JE, Hetzel J (1993) A genetic map of DNA loci on bovine chromosome 1. Genomics 18, 602-608. doi:10.1016/ S0888-7543(05)80362-3

Barendse W, Armitage SM, Kossarek LM, Shalom A, Kirkpatrick BW, et al. (1994) A genetic linkage map of the bovine genome. Nature Genetics 6, 227-235. doi:10.1038/ng0394-227

Barendse W, Vaiman D, Kemp SJ, Sugimoto Y, Armitage SM, et al. (1997) A medium-density linkage map of the bovine genome. Mammalian Genome 8, 21-28. doi:10.1007/s003359900340

Barendse W, Bunch R, Thomas M, Armitage S, Baud S, Donaldson N (2004) The TG5 thyroglobulin gene test for a marbling QTL evaluated in feed lot cattle. Australian Journal of Experimental Agriculture 44, 669-674.

Bishop MD, Kappes SM, Keele JW, Stone RT, Sunden SLF, et al. (1994) A genetic linkage map for cattle. Genetics 136, 619-639.

Bouton PE, Harris PV (1972) A comparison of some objective methods used to assess meat tenderness. Journal of Food Science 37, 218-221.

Bouton PE, Harris PV, Shorthose WR (1971) Effect of ultimate pH upon the water-holding capacity and tenderness of mutton. Journal of Food Science 36, 435-438.

Brezinsky L, Kemp SJ, Teale AJ (1993) ILSTS006: a polymorphic bovine microsatellite. Animal Genetics 24, 73.

Chung HY, Davis ME, Hines HC (1999) A DNA polymorphism of the bovine calpastatin gene detected by SSCP analysis. Animal Genetics 30, 80. doi:10.1046/j.1365-2052.1999.00323-20.x 
Davis GP, Hetzel DJS, Corbet NJ, Scacheri S, Lowden S, Renaud J, Mayne C, Stevenson R, Moore SS, Byrne K (1998) The mapping of quantitative trait loci for birth weight in a tropical beef herd. 6th World Congress of Genetics Applied to Livestock Production 26, 441-444.

Ferguson DM, Bruce HL, Thompson JM, Egan AF, Perry D, Shorthose WR (2001) Factors affecting beef palatability - farmgate to chilled carcass. Australian Journal of Experimental Agriculture 41, 879-892. doi:10.1071/EA00022

Georges M, Nielsen D, Mackinnon M, Mishra A, Okimoto R, et al. (1995) Mapping quantitative trait loci controlling milk production in dairy cattle by exploiting progeny testing. Genetics 139, 907-920.

Green RD, Cockett NE, Tatum JD, O'Connor SF, Hancock DL, Smith GC (1996a) Association of a TaqI calpastatin polymorphism with postmortem measures of beef tenderness in Bos taurus and Bos taurus-indicus steers and heifers Journal of Animal Science 74(Suppl 1), 111.

Green RD, Cockett NE, Tatum JD, Wulf DM, Hancock DL, Smith GC (1996b) Association of a TaqI calpastatin polymorphism with postmortem measures of beef tenderness in Charolais- and Limousin-sired steers and heifers. Journal of Animal Science 74(Suppl 1), 113.

Kappes SM, Keele JW, Stone RT, McGraw RA, Sonstegard TS, Smith TPL, Lopez-Corrales NL, Beattie CW (1997) A secondgeneration linkage map of the bovine genome. Genome Research 7 , 235-249.

Kerr RJ, McLachlan GM, Henshall JM (2005) Use of the EM algorithm to detect QTL affecting multiple-traits in an across half-sib family analysis. Genetics, Selection, Evolution. 37, 83-103.

Koohmaraie M (1996) Biochemical factors regulating the toughening and tenderisation processes of meat. Meat Science 43, 193-201. doi:10.1016/0309-1740(96)00065-4

Kossarek LM, Grosse WM, Finlay O, McGraw RA (1994) Five bovine dinucleotide repeat polymorphisms: RM011, RM012, RM016, RM019 and RM024. Animal Genetics 25, 205-206.

Kuypers R, Kurth LB (1995) Collagen's contribution to meat texture. In 'Meat 95. Proceedings of the Australian Meat Industry research conference'. pp. 11B-18. (McBenny Pty Ltd: Brisbane)

Lander ES, Kruglyak L (1995) Genetic dissection of complex traits: guidelines for interpreting and reporting linkage results. Nature Genetics 11, 241-247. doi:10.1038/ng1195-241

Lonergan SM, Ernst CW, Bishop MD, Calkins CR, Koohmaraie M (1995) Relationship of restriction fragment length polymorphisms (RFLP) at the bovine calpastatin locus to calpastatin activity and meat tenderness. Journal of Animal Science 73, 3608-3612.
Maniatis T, Frisch EF, Sambrook J (1982) 'Molecular cloning, a laboratory manual.' (Cold Spring Harbor Laboratory Press: Cold Spring Harbor, NY)

Moore SS, Barendse W, Berger KT, Armitage SM, Hetzel DJS (1992) Bovine and ovine DNA microsatellites from the EMBL and Genbank databases. Animal Genetics 24, 463-467.

Moore SS, Byrne K, Berger KT, Barendse W, McCarthy F, Womack JE, Hetzel DJS (1994) Characterization of 65 bovine microsatellites. Mammalian Genome 5, 84-90. doi:10.1007/BF00292333

Oddy VH, Harper GS, Greenwood PL, McDonagh MB (2001) Nutritional and developmental effects on the intrinsic properties of muscles as they relate to the eating quality of beef. Australian Journal of Experimental Agriculture 41, 921-942. doi:10.1071/ EA00029

Orita M, Iwahana H, Kanazawa H, Hayashi K, Sekiya T (1989) Detection of polymorphisms of human DNA by gel electrophoresis as single-strand conformation polymorphisms. Proceedings of the National Academy of Sciences USA 86, 2766-2770.

Page BT, Casas E, Heaton MP, Cullen NG, Hyndman DL, et al. (2002) Evaluation of single-nucleotide polymorphisms in CAPN1 for association with meat tenderness in cattle. Journal of Animal Science 80, 3077-3085.

Palmer BR, Hickford JGH, Bickerstaffe R (1997) A candidate gene approach to animal quality traits. Proceedings of the New Zealand Society of Animal Production 57, 294-296.

Stone RT, Pulido JC, Duyk GM, Kappes SM, Keele JW, Beattie CW (1995) A small-insert bovine genomic library highly enriched for microsatellite repeat sequences. Mammalian Genome 6, 714-724. doi: $10.1007 / \mathrm{BF} 00354294$

Vaiman D, Mercier D, Moazami-Goudarzi K, Eggen A, Ciampolini R, et al. (1994) A set of 99 cattle microsatellites: characterisation, synteny mapping, and polymorphism. Mammalian Genome 5, 288-297. doi:10.1007/BF00389543

Whipple G, Koohmaraie M, Dikeman ME, Crouse JD, Hunt MC, Klemm RD (1990) Evaluation of attributes that affect longissimus muscle tenderness in Bos taurus and Bos indicus cattle. Journal of Animal Science 68, 2716-2728.

Received 28 June 2005, accepted 15 December 2005 